\title{
Enhanced tumor cures after Foscan photodynamic therapy combined with the ceramide analog LCL29. Evidence from mouse squamous cell carcinomas for sphingolipids as biomarkers of treatment response
}

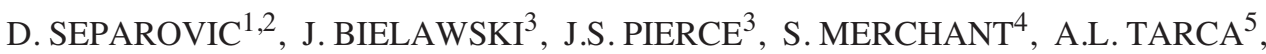 \\ G. BHATTI $^{1}$, B. OGRETMEN ${ }^{3}$ and M. KORBELIK ${ }^{4}$
}

\begin{abstract}
${ }^{1}$ Department of Pharmaceutical Sciences, Eugene Applebaum College of Pharmacy and Health Sciences, Wayne State University, 259 Mack Ave.; ${ }^{2}$ Karmanos Cancer Institute, 4100 John R, Wayne State University, Detroit, MI 48201; ${ }^{3}$ Department of Biochemistry and Molecular Biology, Medical University of South Carolina, 173 Ashley Ave, Charleston, SC 29425, USA; ${ }^{4}$ British Columbia Cancer Agency, 675 West 10th Ave., Vancouver, BC V5Z 1L3, Canada; ${ }^{5}$ Department of Computer Science, Wayne State University, 5143 Cass Ave., Detroit, MI 48202, USA
\end{abstract}

Received July 8, 2010; Accepted August 2, 2010

DOI: $10.3892 /$ ijo. 2010.863

\begin{abstract}
To improve anticancer therapeutic success of photodynamic therapy (PDT), combination treatments represent a viable strategy. Sphingolipid analogs combined with anticancer drugs can enhance tumor response. We have shown that LCL29, a C6-pyridinium ceramide, promotes therapeutic efficacy of Photofrin-PDT in mouse SCCVII squamous cell carcinoma tumors. The long-term effect of the combination PDT + LCL29 is unknown. In this study we used the same model to test the long-term curative potential of Foscan-PDT + LCL29. We show that treatment of SCCVII tumors with the combination led to enhanced long-term tumor cure compared to PDT alone. LCL29 itself did not prevent tumor growth. All treatments triggered early increases in tumor-associated C16-ceramide, C18-ceramide, dihydrosphingosine, and global levels of dihydroceramides. PDT-evoked increases in tumorassociated sphingosine-1-phosphate and dihydrosphingosine1-phosphate remained elevated or were attenuated after the combination, respectively; in contrast, LCL29 had no effect on these two sphingolipids. Our data demonstrate that adjuvant LCL29 improves PDT long-term therapeutic
\end{abstract}

Correspondence to: Dr D. Separovic, Department of Pharmaceutical Sciences, Wayne State University, 259 Mack Ave., Detroit, MI 48201, USA

E-mail: dseparovic@wayne.edu

Key words: C6-pyridinium ceramide, dihydroceramide, dihydrosphingosine, dihydrosphingosine-1-phosphate, ceramide, Foscan, photodynamic therapy, sphingolipids, sphingosine-1-phosphate, squamous cell carcinoma efficacy, implying translational potential of the combination. Furthermore, our findings indicate that changes in the sphingolipid profile might serve as predictive biomarkers of tumor response to treatments.

\section{Introduction}

Photodynamic therapy (PDT) is a clinically established treatment modality for effective eradication of malignancies. In PDT, a light-absorbing agent (photosensitizer) is activated by highly-focused laser light to trigger oxidative stress and destruction of a cellular target $(1,2)$. PDT itself, however, can be ineffective with some tumors $(3,4)$. To overcome inefficiency of PDT alone to eradicate tumors, combined treatments become a necessary option.

Sphingolipids (SLs) are not only building blocks of cell membranes but have been shown to regulate a variety of biological functions (5). Ceramide has been implicated in tumor-suppressor functions, whereas sphingosine-1-phosphate (S1P) can be considered a tumor-promoting agent (6). Relative amounts of dihydrosphingosine-1-phosphate (DHS1P) and S1P may be important during physiological and pathological processes (7). Besides their functional significance, SLs have been proposed as biomarkers for new drug development, in particular in preclinical studies (8).

Targeting SLs has been used for designing new anticancer drugs $(9,10)$. Water-soluble cationic pyridinium ceramide analogs, e.g., C6-pyridinium ceramides LCL29 (Fig. 1A) and LCL124; C16-pyridinium ceramide LCL30, have been synthesized (11). Similar to other pyridinium ceramide analogs, LCL29 accumulates preferentially in negativelycharged mitochondria of various cancer cell lines (11-13). Mitochondrial targeting for cancer treatment is important because cancer cells tend to have mitochondria with more negative mitochondrial membrane potential (14). Pyridinium 
ceramide analogs have been shown to act as effective anticancer agents, alone or in combination with standard chemotherapy $(10,12,13,15-17)$.

Using LCL29 $(11,18)$, in combination with PhotofrinPDT, we have shown moderately improved in vivo response of mouse SCCVII squamous carcinomas (19). However, the long-term effect of the combination PDT + LCL29 is unknown. The goals of the present study were to determine: i) the levels of LCL29 in tumor and surrounding muscle; ii) the signature effects of PDT, with or without LCL29, on the SL profile, and iii) the long-term therapeutic effect of the combination PDT + LCL29. SLs that were analyzed by mass spectrometry (MS) are depicted in Fig. 1B relative to the de novo ceramide pathway. Others (20-25) and we (19,26-30) have shown the involvement of the de novo ceramide pathway in response to anticancer therapeutics, including PDT, in vitro and in vivo. Foscan, a photosensitizer more potent than Photofrin (31), and clinically proven effective for PDT of head and neck cancers (32), was chosen for these investigations. Syngeneic mouse SCCVII squamous cell carcinoma model, a recognized mouse model for human head and neck cancers (33), was used since intact immune system is a key to PDT therapeutic success $(1,2)$.

\section{Materials and methods}

Tumor model and PDT treatment. As described previously $(33,34)$, SCCVII squamous carcinoma cells $\left(1 \times 10^{6}\right)$ were implanted subcutaneously in female syngeneic $\mathrm{C} 3 \mathrm{H} / \mathrm{HeN}$ mice in the sacral lower region on their back. The tumors were allowed to grow until they reached a size of $6-8 \mathrm{~mm}$ in largest diameter (7-10 days). The animals were divided into groups for various treatments. Animals were sacrificed when tumor size was $15 \mathrm{~mm}$ in diameter. For PDT, mice received intraperitoneal injection of Foscan (m-tetrahydroxyphenylchlorin, mTHPC; Biolitec AG, Edinburgh, UK; $0.1 \mathrm{mg} / \mathrm{kg}$ ). Foscan stock solution $(2 \mathrm{mg} / \mathrm{ml})$ was prepared in a mixture of ethanol:polyethyleneglycol400:water $(2 / 3 / 5, \mathrm{v} / \mathrm{v})$. After $24 \mathrm{~h}$, tumors were irradiated (power density, $80-90 \mathrm{~mW} / \mathrm{cm}^{2} ; \lambda$, $650 \pm 10 \mathrm{~nm}$; fluence, $50 \mathrm{~J} / \mathrm{cm}^{2}$ ), with light produced by an FBQTH high throughput illuminator (Sciencetech, London, ON, Canada). During irradiation animals were kept restrained unanesthetized in metal holders exposing their backs. Thereafter the mice were monitored for tumor regrowth up to 90 days. At that time the mice without visible or palpable tumor were considered cured. The ceramide analog D-erythro2-N-[6'-(1"'-pyridinium)-hexanoyl]-sphingosine bromide (LCL29, or C6-pyridinium ceramide) was purchased from Avanti Polar Lipids (Alabaster, AL, USA). For experiments, LCL29 was dissolved in distilled water $(16 \mathrm{mg} / \mathrm{ml})$ and injected intraperitoneally $(80 \mathrm{mg} / \mathrm{kg}) 24 \mathrm{~h}$ prior to PDT or immediately following PDT. The procedures with mice were approved and overseen by the Animal Care Committee of the University of British Columbia.

Measurement of SLs by electrospray ionization/double MS. Mice were sacrificed and tumors collected for MS at $4 \mathrm{~h}$ after PDT. As reported previously $(26,27)$ following extraction, SLs were separated by high performance liquid chromatography, introduced to electrospray ionization source and then analyzed
A. Structure of LCL29 (C6-Pyridinium Ceramide)

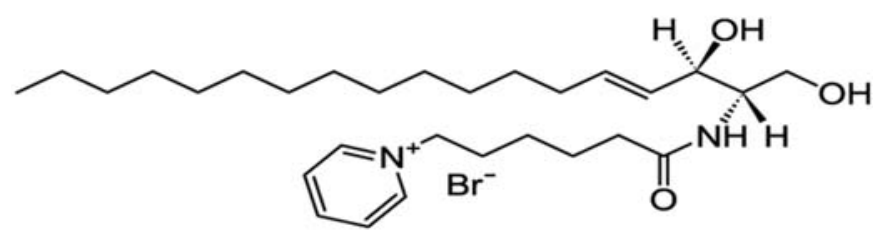

\section{B. De novo ceramide metabolism}

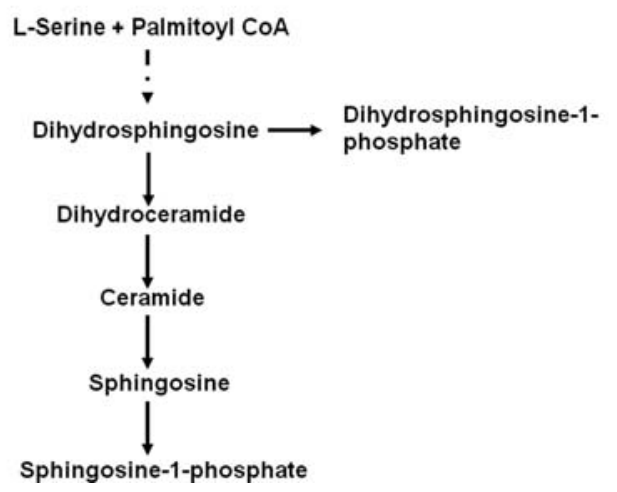

Figure 1. Structure of LCL29 (A). De novo ceramide metabolism (B).

by double MS using TSQ 7000 triple quadrupole mass spectrometer (Thermo Fisher Scientific, San Jose, CA, USA), which allows simultaneous measurement of various SLs, including ceramides, dihydroceramides (DHceramides), dihydrosphingosine (DHsphingosine), DHS1P, sphingosine, and S1P (35). SL levels were normalized to total protein ( $\mathrm{pmol} / \mathrm{mg})$.

Statistical analysis. The effect of each treatment (LCL29, PDT, and PDT + LCL29) on the levels of SLs (pmol/mg) was estimated using a linear model in which the dependent variable was the $\log 2$-transformed level of a SL, while the independent variable was a treatment. Other comparisons, e.g., untreated vs. other controls (Foscan or light alone control) were performed using the two sample $t$-test for unequal variances. The resulting nominal p-values were corrected for multiple comparisons using the false discovery rate (FDR) algorithm. Significance was inferred using a 5\% threshold on the FDR corrected p-values. Overall changes (e.g., for all ceramides) were tested using a one sample $t$-test based on between groups log ratio of individual SLs. All data analysis was performed using the $\mathrm{R}$ statistical language and environment (www.r-project.org). Difference in survival after PDT and PDT + LCL29 was tested using the survival package in R, which allowed also plotting the survival curves. The survival function was estimated using a Kaplan-Meier estimator and the survival curves thus obtained were compared using the log-rank test.

\section{Results}

LCL29 accumulates in SCCVII tumors. Ceramide analog LCL29 was detected in tumors $28 \mathrm{~h}$ after its systemic administration at the dose of $80 \mathrm{mg} / \mathrm{kg}$ (Fig. 2). The levels 
A. LCL29 accumulates in tumor compared to surrounding muscle

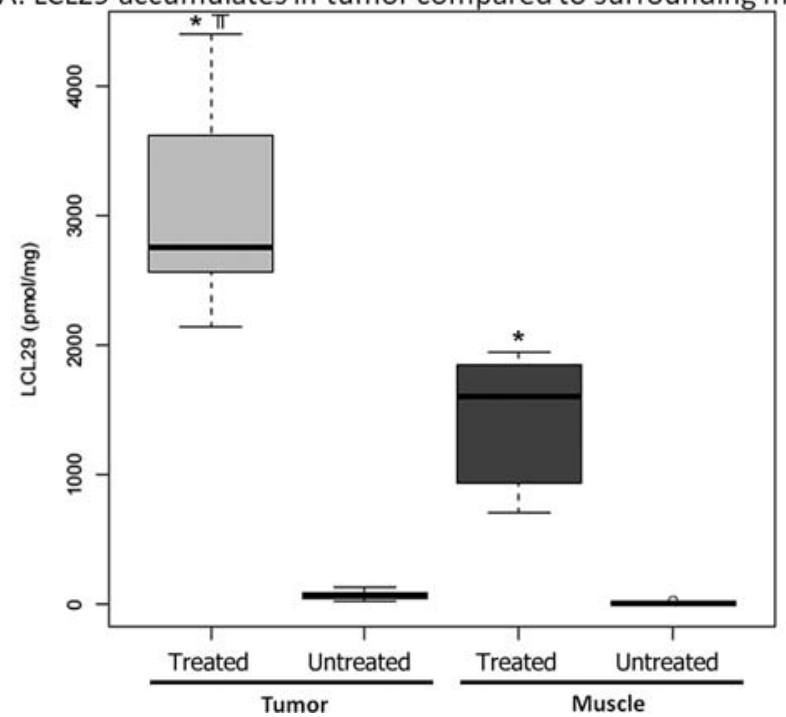

B. LCL29 accumulates in tumor after LCL29 with or without PDT.

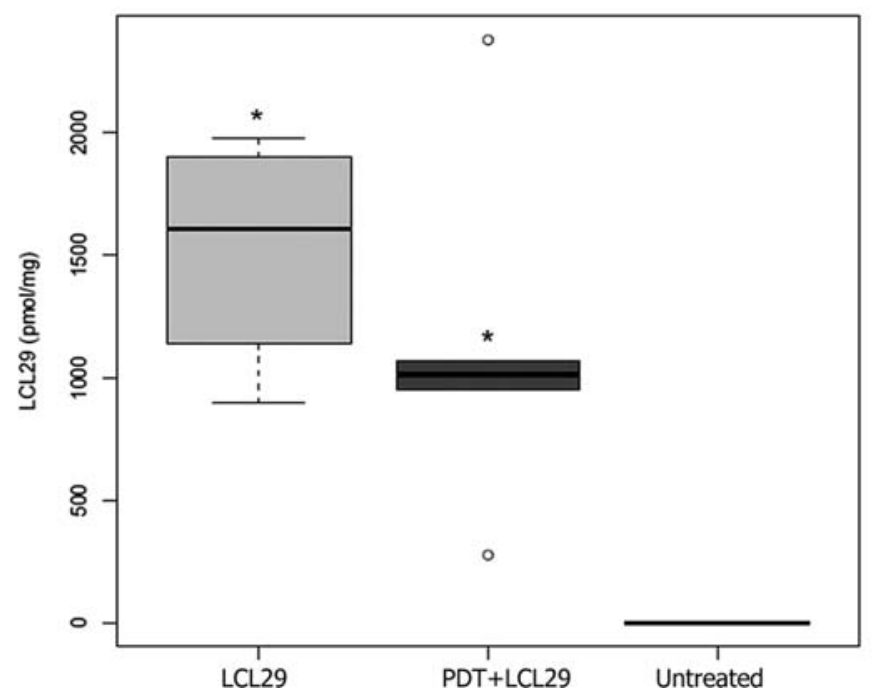

Figure 2. Levels of LCL29 in SCCVII tumors and muscle. (A) LCL29 (80 mg/ $\mathrm{kg}$ ) was injected intraperitoneally. Mice were sacrificed $28 \mathrm{~h}$ later. (B) For PDT + LCL29, Foscan $(0.1 \mathrm{mg} / \mathrm{kg})$ and LCL29 $(80 \mathrm{mk} / \mathrm{kg})$ were injected intraperitoneally $24 \mathrm{~h}$ prior to exposure of SCCVII-bearing mice to light. Mice were sacrificed $4 \mathrm{~h}$ after PDT. Following treatments tumors (A and B) and surrounding muscles (A) were excised and their homogenates were processed for MS analysis. The Y-axis shows tissue-associated LCL29 levels expressed as pmol per mg of total protein. The boxes contain $50 \%$ of the data and the median value is shown as the horizontal thick line within the box. The whiskers extend to the most extreme data point (minimum and maximum value), which is no more than 1.5 times the interquartile range from the box. Significance $(\mathrm{p}<0.05)$ between treated (PDT or PDT + LCL29) and untreated groups was determined by $t$-test on log transformed actual values of LCL29 levels (pmol/mg) and is indicated by asterisks. Significance $(\mathrm{p}<0.05)$ between tumor and muscle post-LCL29 was determined by $t$-test and is indicated by $\pi$. There was no difference in endogenous LCL29 levels after administration of PDT + LCL29 compared to LCL29 alone.

of LCL29 in tumors were 2.16-times higher than in the surrounding muscle $(\mathrm{p}<0.05$; Fig. $2 \mathrm{~A})$. Tumor levels of LCL29 were not significantly different between LCL29 alone and PDT + LCL29 group (Fig. 2B). Mouse tumor levels of LCL29 are comparable to those of its analog LCL124 (13) in
Table I. The effect of PDT, LCL29 and PDT+LCL29 on the tumor SL profile. ${ }^{\mathrm{a}}$

\begin{tabular}{|c|c|c|c|}
\hline & PDT & LCL29 & PDT+LCL29 \\
\hline C14-Ceramide & $1.60^{*}$ & 1.34 & 1.07 \\
\hline C16-Ceramide & 1.63 & $2.36^{*}$ & $1.92^{*}$ \\
\hline C18-Ceramide & 2.33 & $1.95^{*}$ & 2.19 \\
\hline C18:1-Ceramide & 0.97 & 0.86 & 0.88 \\
\hline C20-Ceramide & 1.02 & 0.84 & 0.87 \\
\hline C20:1-Ceramide & 0.80 & 0.48 & 0.98 \\
\hline C22-Ceramide & 1.04 & 1.00 & 1.08 \\
\hline C22:1-Ceramide & 1.00 & 1.24 & 1.08 \\
\hline C24-Ceramide & 0.75 & 0.89 & 0.77 \\
\hline C24:1-Ceramide & 0.80 & 0.92 & 0.87 \\
\hline C26-Ceramide & 0.78 & 0.77 & 0.72 \\
\hline C26:1-Ceramide & 0.59 & 0.66 & 0.68 \\
\hline Global fold change & 1.03 & 1.01 & 1.02 \\
\hline C14-DHCeramide & 2.86 & 0.65 & 1.80 \\
\hline C16-DHCeramide & 6.68 & 1.57 & 3.92 \\
\hline C18-DHCeramide & 5.38 & 1.60 & 3.58 \\
\hline C18:1-DHCeramide & 3.81 & 1.65 & 2.76 \\
\hline C20-DHCeramide & 1.72 & 0.76 & 1.05 \\
\hline C20:1-DHCeramide & 0.97 & 1.57 & 1.82 \\
\hline C22-DHCeramide & 2.73 & 1.39 & 1.51 \\
\hline C22:1-DHCeramide & 2.84 & 1.51 & 2.57 \\
\hline C24-DHCeramide & 2.46 & 1.22 & 1.39 \\
\hline C24:1-DHCeramide & 3.13 & 1.60 & 1.88 \\
\hline C26-DHCeramide & 1.13 & 1.77 & 1.31 \\
\hline C26:1-DHCeramide & 2.18 & 1.10 & 1.37 \\
\hline Global fold change & 2.60 & 1.28 & 1.91 \\
\hline DHSphingosine & 4.78 & 2.47 & 2.30 \\
\hline Sphingosine & 1.12 & 1.58 & 1.07 \\
\hline $\mathrm{S} 1 \mathrm{P}$ & 2.11 & 1.16 & 2.20 \\
\hline DHS1P & 11.01 & 1.24 & 5.12 \\
\hline
\end{tabular}

a The data are shown as ratios compared to untreated controls. Significant values at $\mathrm{p}<0.05$ and $\mathrm{p}<0.1$ are shown in bold and marked by asterisks, respectively.

a xenograft model of human head and neck squamous carcinomas.

Effects of Foscan-PDT, with or without LCL29, on ceramides of SCCVII tumors. Tumors were treated with a therapeutic dose of Foscan-PDT, LCL29, or the combination. Tumor SL profile was identified by MS at $4 \mathrm{~h}$ after irradiation. This time point was chosen since previously we showed at $4 \mathrm{~h}$ post-PDT significant changes in the in vivo SL profile (19). Globally, there was a small decrease in ceramides postFoscan and light alone (28 and 13\%, respectively). Similar global trends were observed for DHceramides. When ceramide and DHceramide levels from PDT-treated group 
A. C18-Ceramide tumor levels are increased after treatments.

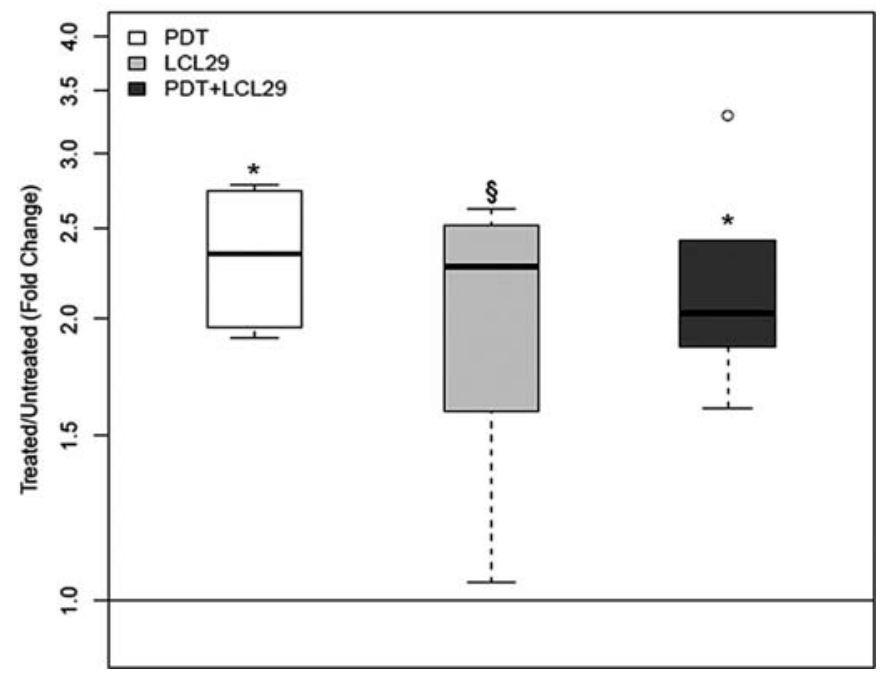

C. The effects of treatments on global ceramides and DHceramides

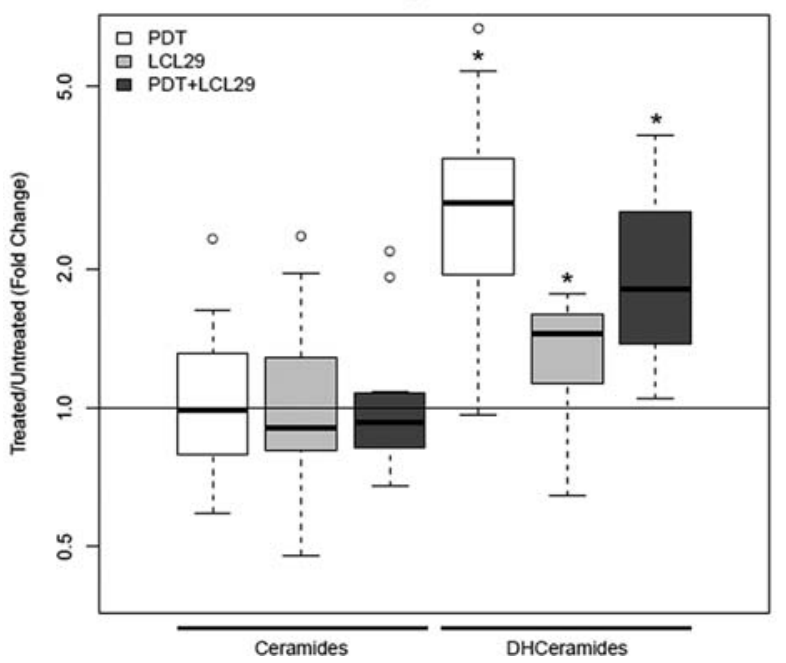

B. DHceramide tumor levels are increased after treatments.

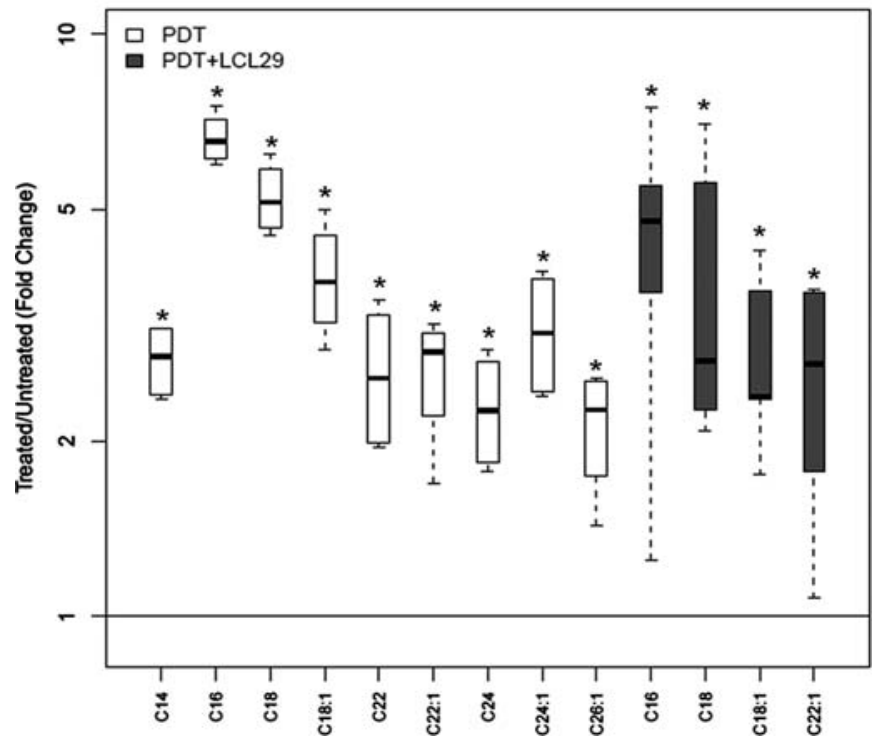

D. The effects of treatments on tumor levels of other SLS.

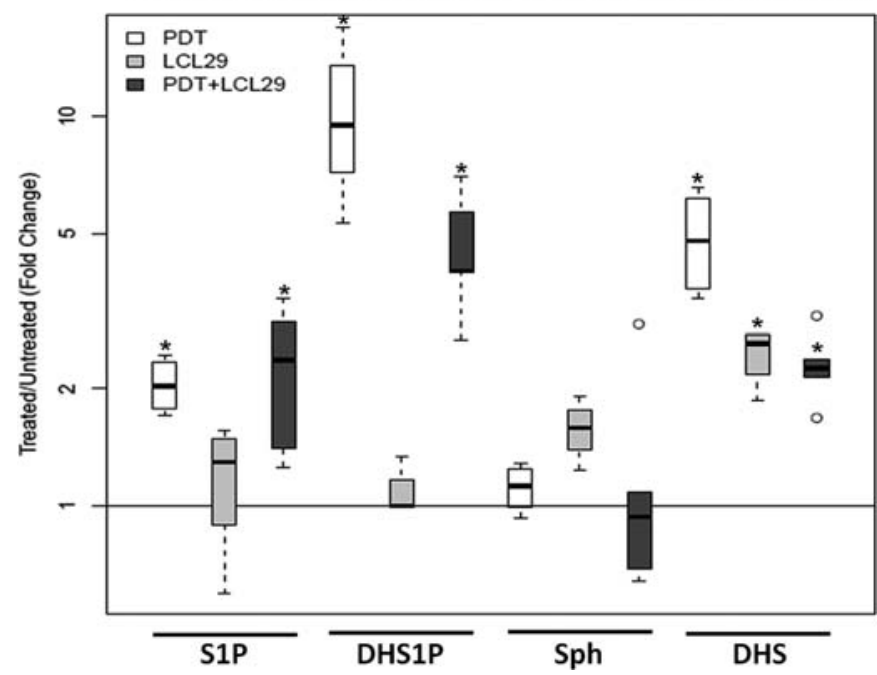

Figure 3. Distinct signature effects on the in vivo SL profile of Foscan-PDT with or without LCL29. Foscan (0.1 mg/kg) and/or LCL29 (80 mk/kg) were injected intraperitoneally $24 \mathrm{~h}$ prior to exposure of SCCVII-bearing mice to light $\left(50 \mathrm{~J} / \mathrm{cm}^{2}\right)$. Mice were sacrificed $4 \mathrm{~h}$ after PDT. Each group consisted of $4-6$ mice. The effects of PDT, LCL29, and the combination on ceramides, DHceramides, and other SLs are shown in panels A, B, and D, respectively. The data were plotted as the ratios of SL levels in treated $v s$. untreated tumors. For Y-axis, a log scale was used. Significance at $\mathrm{p}<0.05$ and $\mathrm{p}<0.1$ is indicated by asterisk and §, respectively. (C) Global levels of ceramides and DHceramides after treatments are shown. (D) Sph, sphingosine; DHS, DHsphingosine.

were compared with Foscan alone (not shown) or untreated groups, they were similar. Resting levels of other SLs were not significantly affected after Foscan or light alone (not shown).

The effects of treatments on ceramide levels are shown in Table I and Fig. 3A and C. None of the treatments had any effect on global levels of ceramide. This is in agreement with our previous finding that after Photofrin-PDT there was no effect on global tumor ceramide levels (19). In contrast to our previous findings, in the present study the levels of C16- and C18-ceramide were elevated after each treatment. In the previous study the photosensitizer Photofrin alone had strong effects on the SL profile (19), which could account for lowering signal-to-noise ratio.

Effects of Foscan-PDT, with or without LCL29, on DHceramides of SCCVII tumors. The effects of treatments on
DHceramide levels are shown in Table I; Fig. 3B and C. Following PDT, the overall tumor DHceramide levels were increased 2.60-fold. The greatest increases of 6.68- and 5.38-fold were observed for C16- and C18-DHceramide, respectively. The levels of C14-, C18:1-, C22-, C22:1-, C24-, C24:1 - and C26:1-DHceramide were also significantly increased. Analysis of the effects of LCL29 alone on global tumor DHceramide levels showed a 1.28-fold increase. Following treatment with the combination, the levels of C16-, C18-, C18:1- and C22:1-DHceramide were significantly increased. Moreover, there was a significant, 1.91-fold global increase in DHceramides after the combination compared to untreated controls. Similarly, we observed both global and selective increase in DHceramides after Photofrin-PDT (19). Overall, the data demonstrate global increases in DHceramides after all treatments and selective increases after PDT alone and the combination. 


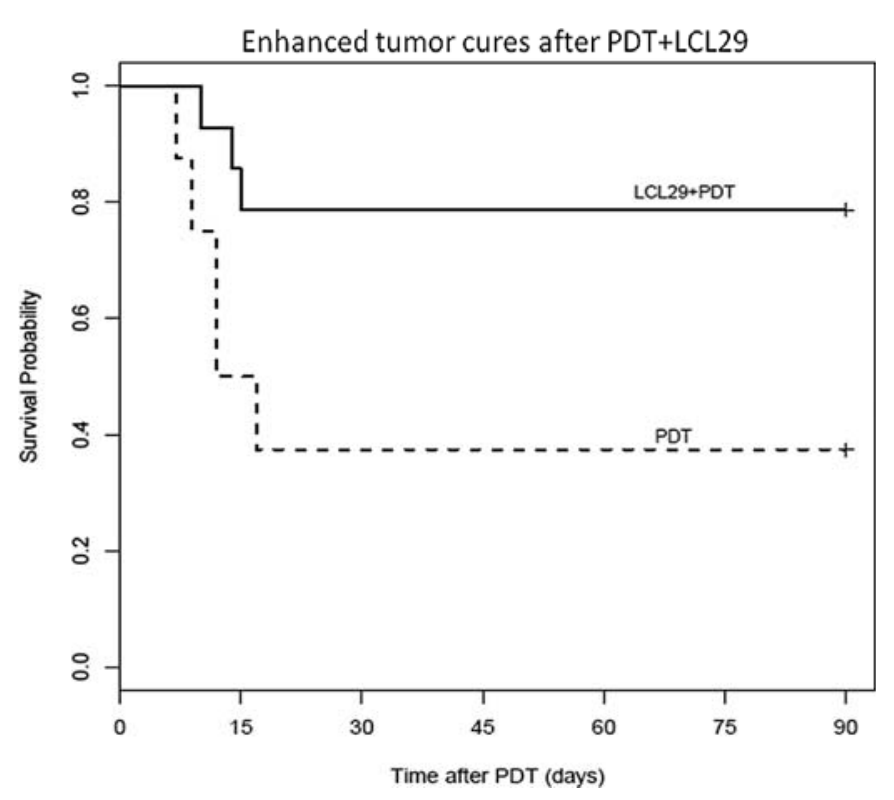

Figure 4. Adjuvant LCL29 improves cures of tumors treated with FoscanPDT. SCCVII tumors were treated with either PDT (see legend for Fig. 2), LCL29 $(80 \mathrm{mg} / \mathrm{kg}$, intraperitoneally), or their combination (with LCL29 administered either at the time of Foscan injection or immediately after irradiation for PDT). A control group and PDT only group received vehicle injections as in the LCL29 treatment protocol. Each experimental group consisted of 8 mice. After administering treatments, the mice were monitored up to 90 days for signs of tumor growth. The mice without palpable/visible tumors were considered 'tumor-free'. A statistically significant difference $(\mathrm{p}<0.04)$ was found between the Kaplan-Meier survival curves for the combination (from both protocols) and PDT alone.

Effects of Foscan-PDT, with or without LCL29, on other SLs of SCCVII tumors. The effects of treatments on the levels of other SLs are shown in Table I and Fig. 3D. Tumor levels of DHsphingosine, a precursor of DHceramide and DHS1P, were increased after all treatments. LCL29 alone had no significant effect on other SLs. Notably, the levels of DHS1P, a product of DHsphingosine, were increased 11-fold after PDT. An attenuated but significant increase (5.12-fold) of DHS1P was observed after the combination. S1P levels were increased 2.11-fold after PDT, and the effect was maintained after the combination. Tumor-associated levels of sphingosine were not significantly changed after any of treatments. Taken together, the data show that tumor levels of DHsphingosine, DHS1P and S1P are modulated following administration of treatments.

Enhanced tumor cures after Foscan-PDT + LCL29. To test whether LCL29 can improve long-term tumor cures after PDT, SCCVII tumors were treated with a moderately therapeutic dose of Foscan-PDT and a non-toxic dose of LCL29 (13). LCL29 was administered intraperitoneally either $24 \mathrm{~h}$ before PDT or immediately after PDT. Treatment with LCL29 alone produced no detectable effect on tumors, as they continued to grow at the similar rate as untreated tumors (not shown). Tumor cure rates were: $37.5 \%$ with PDT only group; $83.3 \%$ with LCL29 given one day before PDT, and 75.0\% with LCL29 given immediately after PDT. Since there was no apparent difference in the outcome between the two protocols for the combination, the data from the two protocols were combined and compared to PDT (Fig. 4). Using the log-rank test, the comparison of the Kaplan-Meier survival curves for PDT and the combination (from both protocols) yielded a Chi-square statistic of 4.2 with one degree of freedom $(\mathrm{p}<0.05)$. Hence, the results from both protocols using the combination demonstrate enhanced tumor cures compared to PDT alone. These are important data showing that PDT long-term tumor response is promoted by an SL-modulating agent.

\section{Discussion}

Since classic single treatment modalities can fail, combined therapies for cancer hold a great potential. Although PDT alone can effectively irradicate local malignancies, including head and neck squamous cell carcinomas (36), tumor recurrence does occur $(3,4)$. The present study demonstrates for the first time that there is a long-term therapeutic benefit of the combination PDT + LCL29.

There is emerging evidence in support of predictive biomarkers that are used to determine tumor response to therapy (37). We observed that PDT-evoked increase (11-fold) in tumor-associated DHS1P was attenuated (5.12-fold) after the combination, whereas LCL29 had no effect on DHS1P. S1P levels were elevated after PDT or the combination, but not after LCL29. There was a trend toward greater increases in global levels of DHceramides after PDT or the combination than after LCL29. Thus, the data support the idea that there is correlation between patterns of responses of certain SLs to treatments and therapeutic outcome.

Increases in DHceramides and DHsphingosine after treatments, support the notion that the de novo ceramide pathway is a target. Others (20-25) and we (26-30) have shown the involvement of de novo SLs in response to anticancer therapeutics. Here we observed, following PDT or the combination, a significant increase in C16- and C18-DHceramide, the products of DHceramide synthase 6 and 1, respectively. DHceramide synthase 1 has been associated with chemotherapy-triggered killing of human head and neck squamous cell carcinomas (23). In contrast, DHceramide synthase 6 has been shown to have an antiapoptotic role in the same model (38). The role of DHceramide synthases in response to PDT + LCL29 will be addressed in future experiments.

The increases in S1P after PDT or the combination may not have been anticipated, since increased levels of S1P are associated with a survival response (39). Notably, the increases in DHS1P were far greater than those of S1P. It has been suggested that the relative amount of DHS1P vs. S1P may be important during physiological and pathological processes (7).

To sum up, the present study demonstrates for the first time that: i) the levels of LCL29 are greater in tumor than in surrounding muscle; ii) tumor-associated SLs respond early to treatments, and iii) there is the long-term therapeutic benefit of the combination PDT + LCL29. Our findings have the following implications: i) changes in the SL profile might serve as predictive markers of tumor response to treatments, ii) targeting the de novo ceramide pathway might be a strategy for drug development to advance PDT tumor response, and iii) the combination PDT + LCL29 might have translational potential. 


\section{Acknowledgements}

This work was supported by U.S. Public Health Service Grant R01 CA77475 from the National Cancer Institute (D.S.), and partly by the grant DE016572 from the National Institute of Dental and Craniofacial Research (B.O.). The MSrelated work was done at the Lipidomics Shared Resource (Department of Biochemistry and Molecular Biology, Medical University of South Carolina, Charleston), a facility supported by the U.S. Public Health Service Grant C06 RR018823 from the Extramural Research Facilities Program, National Center for Research Resources.

\section{References}

1. Dougherty TJ, Gomer CJ, Henderson BW, Jori G, Kessel D, Korbelik M, Moan J and Peng Q: Photodynamic Therapy: Review. J Natl Cancer Inst 90: 889-902, 1998.

2. Castano AP, Mroz P and Hamblin MR: Photodynamic therapy and anti-tumour immunity. Nat Rev Cancer 6: 535-545, 2006.

3. Wilson JJ, Jones H, Burock M, Smith D, Fraker DL, Metz J, Glatstein E and Hahn SM: Patterns of recurrence in patients treated with photodynamic therapy for intraperitoneal carcinomatosis and sarcomatosis. Int J Oncol 24: 711-717, 2004.

4. Rhodes LE, de Rie M, Enstrom Y, Groves R, Morken T, Goulden V, Wong GA, Grob JJ, Varma S and Wolf P: Photodynamic therapy using topical methyl aminolevulinate vs. surgery for nodular basal cell carcinoma: results of a multicenter randomized prospective trial. Arch Dermatol 140: 17-23, 2004.

5. Hannun YA and Obeid LM: Principles of bioactive lipid signalling: lessons from sphingolipids. Nat Rev Mol Cell Biol 9: 139-150, 2008.

6. Ogretmen B and Hannun YA: Biologically active sphingolipids in cancer pathogenesis and treatment. Nat Rev Cancer 4: 604-616, 2004

7. Bu S, Kapanadze B, Hsu T and Trojanowska M: Opposite effects of dihydrosphingosine 1-phosphate and sphingosine 1-phosphate on transforming growth factor-beta/Smad signaling are mediated through the PTEN/PPM1A-dependent pathway. J Biol Chem 283: 19593-19602, 2008

8. Mielke MM and Lyketsos CG: Alterations of the sphingolipid pathway in Alzheimer's disease: New biomarkers and treatment targets? Neuromolecular Med, June 23, 2010 (Epub ahead of print)

9. Fox TE, Finnegan CM, Blumenthal R and Kester M: The clinical potential of sphingolipid-based therapeutics. Cell Mol Life Sci 63: 1017-1023, 2006.

10. Zeidan YH and Hannun YA: Translational aspects of sphingolipid metabolism. Trends Mol Med 13: 327-336, 2007.

11. Szulc ZM, Bielawski J, Gracz H, Gustilo M, Mayroo N, Hannun YA, Obeid LM and Bielawska A: Tailoring structurefunction and targeting properties of ceramides by site-specific cationization. Bioorg Med Chem 14: 7083-7104, 2006.

12. Novgorodov SA, Szulc ZM, Luberto C, Jones JA, Bielawski J, Bielawska A, Hannun YA and Obeid LM: Positively charged ceramide is a potent inducer of mitochondrial permeabilization. J Biol Chem 280: 16096-16105, 2005.

13. Senkal CE, Ponnusamy S, Rossi MJ, Sundararaj K, Szulc Z, Bielawski J, Bielawska A, Meyer M, Cobanoglu B, Koybasi S, Sinha D, Day TA, Obeid LM, Hannun YA and Ogretmen B: Potent antitumor activity of a novel cationic pyridinium-ceramide alone or in combination with gemcitabine against human head and neck squamous cell carcinomas in vitro and in vivo. J Pharmacol Exp Ther 317: 1188-1199, 2006.

14. Chen LB: Mitochondrial membrane potential in living cells. Annu Rev Cell Biol 4: 155-181, 1988.

15. Rossi MJ, Sundararaj K, Koybasi S, Phillips MS, Szulc ZM, Bielawska A, Day TA, Obeid LM, Hannun YA and Ogretmen B: Inhibition of growth and telomerase activity by novel cationic ceramide analogs with high solubility in human head and neck squamous cell carcinoma cells. Otolaryngol Head Neck Surg 132: 55-62, 2005.

16. Dindo D, Dahm F, Szulc Z, Bielawska A, Obeid LM, Hannun YA, Graf R and Clavien PA: Cationic long-chain ceramide LCL-30 induces cell death by mitochondrial targeting in SW403 cells. Mol Cancer Ther 5: 1520-1529, 2006.
17. Dahm F, Bielawska A, Nocito A, Georgiev P, Szulc ZM, Bielawski J, Jochum W, Dindo D, Hannun YA and Clavien PA: Mitochondrially targeted ceramide LCL-30 inhibits colorectal cancer in mice. Br J Cancer 98: 98-105, 2008.

18. Bielawska A, Bielawski J, Szulc ZM, Mayroo N, Liu X, Bai A, Elojeimy S, Rembiesa B, Pierce J, Norris JS and Hannun YA: Novel analogs of D-e-MAPP and B13. Part 2: signature effects on bioactive sphingolipids. Bioorg Med Chem 16: 1032-1045, 2008.

19. Separovic D, Bielawski J, Pierce JS, Merchant S, Tarca AL, Ogretmen B and Korbelik M: Increased tumour dihydroceramide production after Photofrin-PDT alone and improved tumour response after the combination with the ceramide analogue LCL29. Evidence from mouse squamous cell carcinomas. Br J Cancer 100: 626-632, 2009.

20. Bose R, Verheij M, Haimovitz-Friedman A, Scotto K, Fuks Z and Kolesnick R: Ceramide synthase mediates daunorubicininduced apoptosis: an alternative mechanism for generating death signals. Cell 82: 405-414, 1995.

21. Charles AG, Han TY, Liu YY, Hansen N, Giuliano AE and Cabot MC: Taxol-induced ceramide generation and apoptosis in human breast cancer cells. Cancer Chemother Pharmacol 47: 444-450, 2001.

22. Perry DK, Carton J, Shah AK, Meredith F, Uhlinger DJ and Hannun YA: Serine palmitoyltransferase regulates de novo ceramide generation during etoposide-induced apoptosis. J Biol Chem 275: 9078-9084, 2000.

23. Senkal CE, Ponnusamy S, Rossi MJ, Bialewski J, Sinha D, Jiang JC, Jazwinski SM, Hannun YA and Ogretmen B: Role of human longevity assurance gene 1 and $\mathrm{C} 18$-ceramide in chemotherapy-induced cell death in human head and neck squamous cell carcinomas. Mol Cancer Ther 6: 712-722, 2007.

24. Min J, Mesika A, Sivaguru M, Van Veldhoven PP, Alexander H, Futerman AH and Alexander S: (Dihydro)ceramide synthase 1 regulated sensitivity to cisplatin is associated with the activation of p38 mitogen-activated protein kinase and is abrogated by sphingosine kinase 1. Mol Cancer Res 5: 801-812, 2007.

25. Wang H, Maurer BJ, Liu YY, Wang E, Allegood JC, Kelly S, Symolon H, Liu Y, Merrill AH Jr, Gouaze-Andersson V, Yu JY, Giuliano AE and Cabot MC: N-(4-Hydroxyphenyl)retinamide increases dihydroceramide and synergizes with dimethylsphingosine to enhance cancer cell killing. Mol Cancer Ther 7: 2967-2976, 2008

26. Separovic D, Hanada K, Maitah MY, Nagy B, Hang I, Tainsky MA, Kraniak JM and Bielawski J: Sphingomyelin synthase 1 suppresses ceramide production and apoptosis postphotodamage. Biochem Biophys Res Commun 358: 196-202, 2007.

27. Separovic D, Semaan L, Tarca AL, Awad Maitah MY, Hanada K, Bielawski J, Villani M and Luberto C: Suppression of sphingomyelin synthase 1 by small interference RNA is associated with enhanced ceramide production and apoptosis after photodamage. Exp Cell Res 314: 1860-1868, 2008.

28. Wispriyono B, Schmelz E, Pelayo H, Hanada K and Separovic D: A role for the de novo sphingolipids in apoptosis of photosensitized cells. Exp Cell Res 279: 153-165, 2002.

29. Dolgachev V, Farooqui MS, Kulaeva OI, Tainsky MA, Nagy B, Hanada K and Separovic D: De novo ceramide accumulation due to inhibition of its conversion to complex sphingolipids in apoptotic photosensitized cells. J Biol Chem 279: 23238-23249, 2004.

30. Dolgachev V, Oberley LW, Huang TT, Kraniak JM, Tainsky MA, Hanada K and Separovic D: A role for manganese superoxide dismutase in apoptosis after photosensitization. Biochem Biophys Res Commun 332: 411-417, 2005.

31. Mitra S and Foster TH: Photophysical parameters, photosensitizer retention and tissue optical properties completely account for the higher photodynamic efficacy of meso-tetra-hydroxyphenylchlorin vs. Photofrin. Photochem Photobiol 81: 849-859, 2005.

32. Hopper C, Niziol C and Sidhu M: The cost-effectiveness of Foscan mediated photodynamic therapy (Foscan-PDT) compared with extensive palliative surgery and palliative chemotherapy for patients with advanced head and neck cancer in the UK. Oral Oncol 40: 372-382, 2004.

33. Khurana D, Martin EA, Kasperbauer JL, O'Malley BW Jr, Salomao DR, Chen L and Strome SE: Characterization of a spontaneously arising murine squamous cell carcinoma (SCC VII) as a prerequisite for head and neck cancer immunotherapy. Head Neck 23: 899-906, 2001. 
34. Sun J, Cecic I, Parkins CS and Korbelik M: Neutrophils as inflammatory and immune effectors in photodynamic therapytreated mouse SCCVII tumours. Photochem Photobiol Sci 1: 690-695, 2002

35. Bielawski J, Szulc ZM, Hannun YA and Bielawska A: Simultaneous quantitative analysis of bioactive sphingolipids by high-performance liquid chromatography-tandem mass spectrometry. Methods 39: 82-91, 2006.

36. Biel MA: Photodynamic therapy treatment of early oral and laryngeal cancers. Photochem Photobiol 83: 1063-1068, 2007.
37. Fareed KR, Kaye P, Soomro IN, Ilyas M, Martin S, Parsons SL and Madhusudan S: Biomarkers of response to therapy in oesophago-gastric cancer. Gut 58: 127-143, 2009.

38. Senkal CE, Ponnusamy S, Bielawski J, Hannun YA and Ogretmen B: Antiapoptotic roles of ceramide-synthase-6generated C16-ceramide via selective regulation of the ATF6/ CHOP arm of ER-stress-response pathways. FASEB J 24: 296-308, 2010.

39. Pyne NJ and Pyne S: Sphingosine 1-phosphate and cancer. Nat Rev Cancer 10: 489-503, 2010. 